\title{
A PERTUSSIS EPIDEMIC IN NSW: HOW EPIDEMIOLOGY REFLECTS VACCINATION POLICY
}

\section{Julia Brotherton and Jeremy McAnulty Communicable Diseases Branch NSW Department of Health}

Pertussis has traditionally been considered a disease of young children. In Australia, combined diphtheriatetanus-acellular pertussis (DTPa) vaccine is currently included in the childhood immunisation schedule at ages 2, 4, 6, and 18 months and, since 1994, before school entry at 4-5 years of age. ${ }^{1}$ Prior to 1999 , a formulation that included whole cell pertussis vaccine was used. In NSW, periodic epidemics of pertussis have occurred every 3-4 years, most recently in 1993-1994, 1997-1998 and now in 2000-2002. A seasonal pattern is clearly evident with peak notification rates occurring in spring every year. Even during the inter-epidemic period of 1999 however, pertussis was still common, with around 115 reported cases per month. The most recent epidemic began in May 2000 when over 200 cases were reported. In this article, we examine the distribution of, and shifts in, ages of pertussis cases notified in the three most recent NSW epidemics, in order to understand the observed age distribution of the 2000-2002 epidemic (when there were relatively few cases in children under five years of age and many cases in young adolescents). The implications for vaccination policy are also reviewed.

\section{METHODS}

By law in NSW, cases of pertussis are notified by doctors, hospitals and laboratories, and by schools and childcare facilities. Public health unit staff routinely follow up notifications of pertussis in order to limit the spread of disease by facilitating timely management of cases and contacts, including education, contact tracing and provision of antibiotic prophylaxis to contacts.

For surveillance, pertussis cases are defined as a person with a coughing illness lasting more than 14 days with:

- either paroxysms of cough, inspiratory 'whoop', or post-tussive vomiting without other apparent cause; or

- a positive Bordetella pertussis-specific IgA; or

- an epidemiological link to a laboratory-confirmed case; or

- a clinical specimen that isolates Bordetella pertussis or is identified by polymerase chain reaction (PCR) in a laboratory experienced in the technique.

We reviewed surveillance data from the NSW Notifiable Diseases Database for the period 1991 to 2002 and identified three epidemic periods when case notification rates appeared to rise above expected levels: May 2000 to April 2002 (24 months), May 1997 to April 1998 (12 months), and May 1993 to April 1994 (12 months). We analysed pertussis notifications by date of onset for these periods at 15 January 2003. Annual rates were calculated using mid-year population estimates from the Australian Bureau of Statistics.

\section{RESULTS}

\section{0-2002 epidemic}

Between 1 May 2000 and 30 April 2002, there were 8,337 notified cases (by onset date) of pertussis (63.8 per 100,000 persons per year) (Figure 1). This number compares with 1,565 notifications $(24.4$ per 100,000$)$ for the preceding

\section{FIGURE 1}

NUMBER OF PERTUSSIS NOTIFICATIONS BY MONTH OF ONSET, NSW, 1991-2003

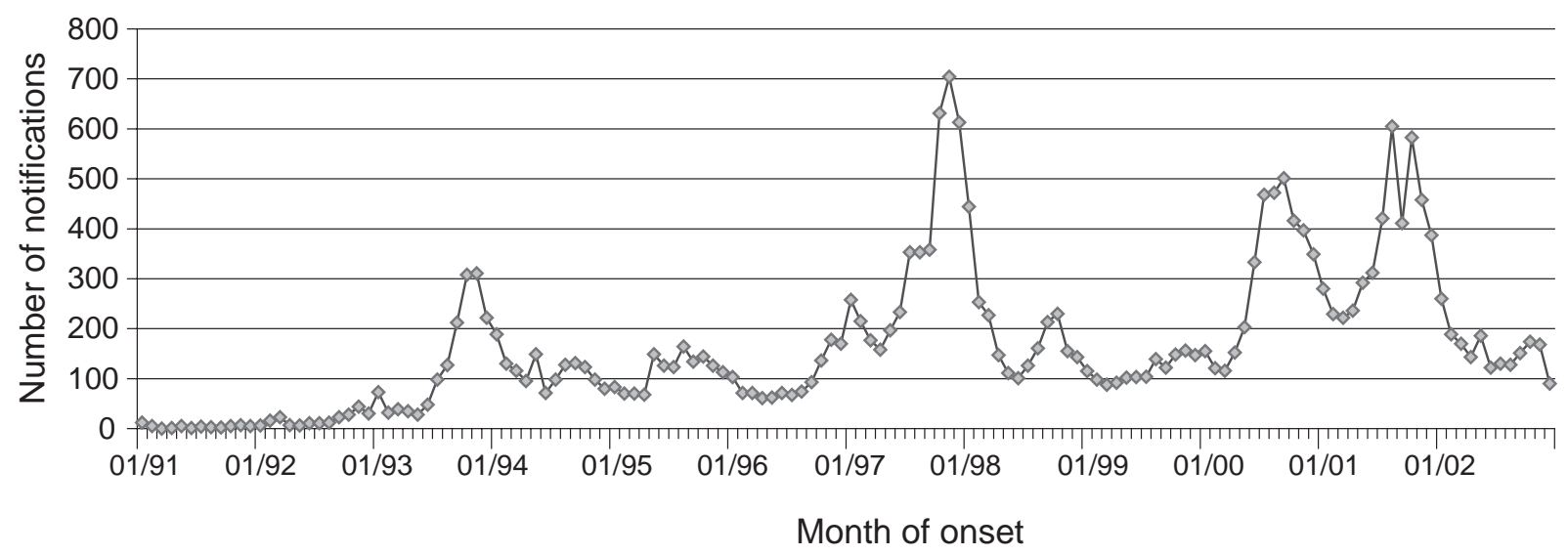

Source: Communicable Diseases Branch, NSW Notifiable Disease Database and ABS population estimates (HOIST), Centre for Epidemiology and Research, NSW Department of Health. 
12 months. There was a slight predominance of female notifications (55.5 per cent). Most notifications were laboratory confirmed (74 per cent) with serology the predominant method (84 per cent).

Over one quarter (27 per cent) of notifications $(N=2223)$ were in the 10 to 14 -year-old age group, with a notification rate of 248.6 per 100,000 per year (Figures 2 and 3). Only eight per cent of notifications were in children 0-4 years of age, eight per cent were in children 5-9 years of age, 10 per cent were in adolescents 15-19 years of age, and the remaining 47 per cent occurred in adults 20 years and over [the overall adult rate was 41.3 per 100,000 per year, peaking by five-year age group in those adults aged 40-44 years (rate 65.3 per 100,000)]. The pertussis notification rate in older adults for those aged 50 years and over was 32.3 per 100,000 per year.

\section{FIGURE 2}

PERTUSSIS NOTIFICATION RATES BY AGE GROUP, NSW. 2000-2002 EPIDEMIC (DATE OF ONSET 1 MAY 2000 TO 30 APRIL 2002)

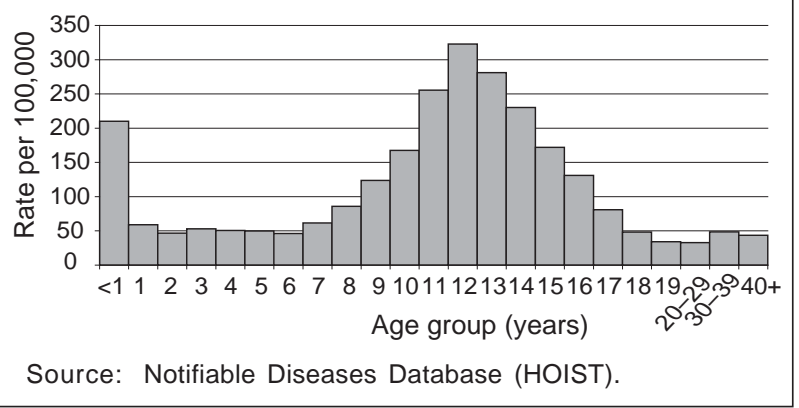

\section{FIGURE 3}

PERCENTAGE OF PERTUSSIS NOTIFICATIONS IN THOSE UNDER 15 YEARS OF AGE $(N=3548)$ BY FIVE-YEAR AGE GROUP, NSW. 2000-2002 EPIDEMIC (DATE OF ONSET 1 MAY 2000 TO 30 APRIL 2002)

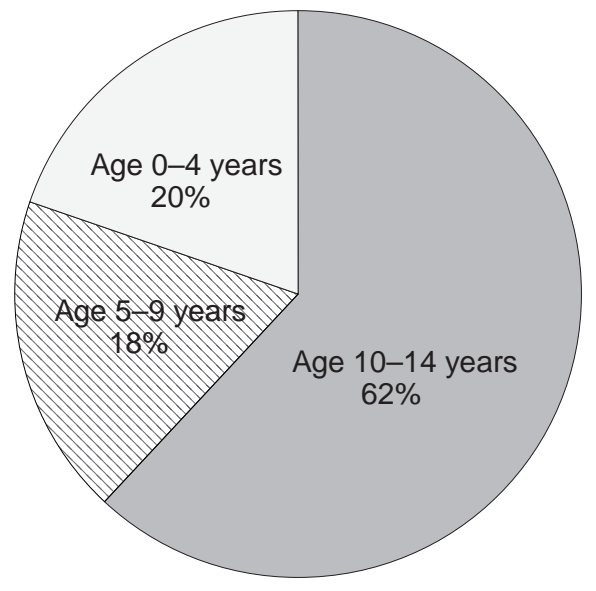

Source: Notifiable Diseases Database (HOIST).
Crude incidence rates fluctuated widely between geographical areas and year of the epidemic (between 8.2 per 100,000 in the remote far west of the state in 2000-2001 and 190.8 per 100,000 in the mixed urban and rural Hunter region in 2000-2001 where the rate subsequently halved in 2001-2002). Over the two-year epidemic period, the highest average incidence rate was reported in the Macquarie Area with 157.5 notifications per 100,000 per year. All areas reported highest rates in 10-14 year olds, ranging from 85.4 per 100,000 per year in the Far West to 608.2 per 100,000 per year in the Macquarie Area. Within this age group, most notifications were in the 11 and 12-year-old age group in year one of the epidemic and in 12 and 13-year-old age group in year two.

There were 345 notified cases in children aged 12 months or younger $(210.0$ per 100,000$)$, with just over half of

\section{FIGURE 4}

PERTUSSIS NOTIFICATION RATES BY AGE GROUP, NSW. 1997-1998 EPIDEMIC (DATE OF ONSET 1 MAY 1997 TO 30 APRIL 1998)

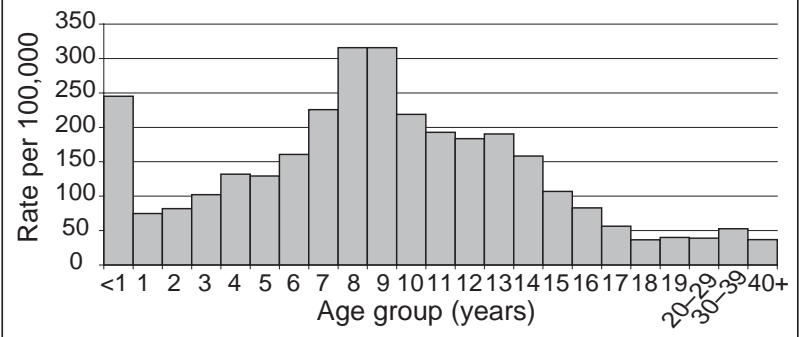

Source: Notifiable Diseases Database (HOIST).

\section{FIGURE 5}

PERCENTAGE OF PERTUSSIS NOTIFICATIONS IN THOSE UNDER 15 YEARS OF AGE $(N=2389)$ BY FIVE-YEAR AGE GROUP, NSW. 1997-1998 EPIDEMIC (DATE OF ONSET 1 MAY 1997 TO 30 APRIL 1998)

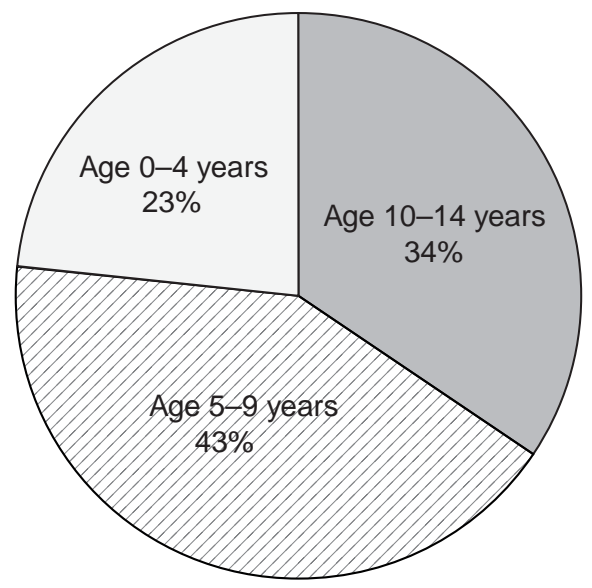

Source: Notifiable Diseases Database (HOIST). 
these ( 51.3 per cent) in those aged three months or younger. At least 107 infants were hospitalised. There was one reported death in a 10 -week old infant. ${ }^{2}$ Box 1 outlines the additional measures taken in response to the epidemic.

\section{BOX 1 \\ ADDITIONAL PUBLIC HEALTH ACTIONS IMPLEMENTED IN 2000-2002 EPIDEMIC}

With increasing notifications of pertussis, the NSW Department of Health convened an expert committee to develop guidelines for doctors and to coordinate a response strategy. After reviewing current evidence, ${ }^{3}$ the committee revised recommendations to reduce the duration of erythromycin treatment and prophylaxis for contacts from 10 to seven days. The shorter duration of treatment was a result of the concern that cost and side-effects of a prolonged course was undermining adherence to its use.

In September 2000, the NSW Department of Health sent all doctors, emergency departments, and school principals in NSW a letter advising of the epidemic and provided a fact sheet for distribution to patients and parents. Doctors were also sent guidelines regarding the diagnosis and management of pertussis. Public health units were emailed monthly pertussis updates that reviewed the latest notification data. Public health units developed local communication and information strategies in addition to the statewide activities. To prevent cases in young infants, fact sheets were distributed to maternity hospitals, and a sticker was designed to be placed upon the cover of the personal health record (or 'blue book') that every newborn baby in NSW receives. This bright yellow sticker (Watch out, whooping cough is about!) warned parents to keep their babies away from anyone with a cough and to have their babies immunised on time.

\section{7-1998 epidemic}

Between 1 May 1997 and 30 April 1998, 4,513 cases were notified (71.9 per 100,000). Of these notifications, 77 per cent were laboratory confirmed with serology the most commonly documented laboratory test ( 94 per cent). Highest notification rates were in five to nine-year-old children (228.8 per 100,000; 22 per cent of notifications) and most notifications in this group were in eight and nine-year-old children. Adolescents aged 10-14 years were the next most commonly affected (188.6 per 100,$000 ; 18$ per cent). Those children under five years of age comprised 12 per cent of notifications and young infants under 12-months of age were notified at a rate of 245.1 per 100,000 (Figures 4 and 5). Two infants died

\section{FIGURE 6}

PERTUSSIS NOTIFICATION RATES BY AGE GROUP, NSW. 1993-1994 EPIDEMIC (DATE OF ONSET 1 MAY 1993 TO 30 APRIL 1994)

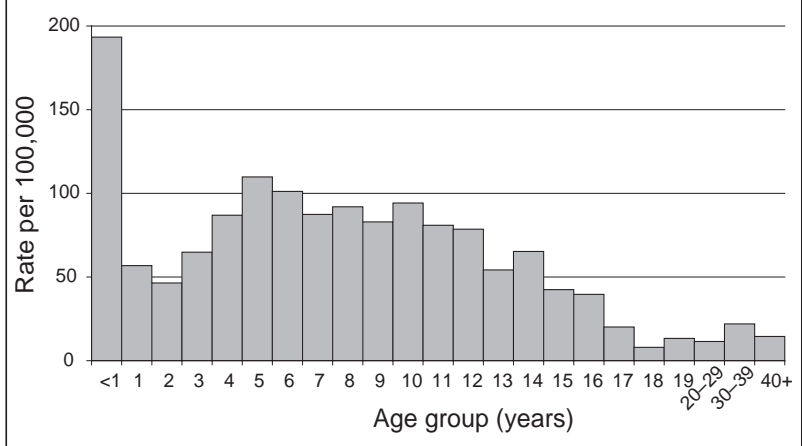

Source: Notifiable Diseases Database and ABS population estimates (HOIST).

\section{FIGURE 7}

PERCENTAGE OF PERTUSSIS NOTIFICATIONS IN THOSE UNDER 15 YEARS OF AGE $(N=1115)$ BY FIVE-YEAR AGE GROUP, NSW. 1993-1994 EPIDEMIC (DATE OF ONSET 1 MAY 1993 TO 30 APRIL 1994)

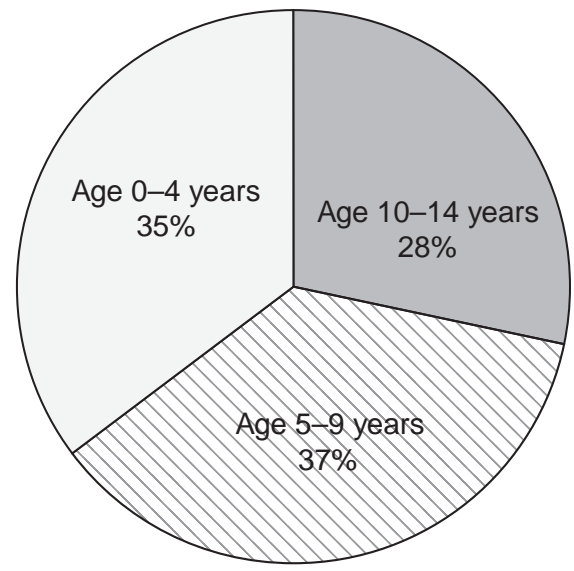

Source: Notifiable Diseases Database (HOIST).

during the epidemic, and four infants had died in the lead up to the epidemic between October 1996 and May 1997.

\section{3-1994 epidemic}

A smaller epidemic occurred between 1 May 1993 and 30 April 1994 when there were 1,884 notifications of pertussis (31.4 per 100,000). Seventy-seven per cent of notifications were confirmed by laboratory testing, with serology the most commonly documented laboratory test (90 per cent). Almost half of the notifications were in children under 10 years (42 per cent) (Figures 6 and 7). By five-year age group, the highest rates were in 5-9-yearold children $(94.7$ per 100,000$)$ followed by the group under five years $(89.8$ per 100,000$)$. Adolescents $10-14$ were notified at a rate of 74.9 per 100,000 (17 per cent of 
notifications). Infants under 12 months of age were notified at a rate of 193.4 per 100,000 . No infant deaths were notified.

\section{DISCUSSION}

We found that the age of notified pertussis cases increased over time in NSW. When comparing pertussis rates with other countries, it must be noted that in NSW, serology (combined with an appropriate clinical history) is the main method resulting in notification of a case. Pertussis IgA serology is quite specific for disease (93-98 per cent). ${ }^{4}$ Rates may appear high compared with countries that do not accept serology-based notifications. However, we believe there remains a substantial under-reporting of pertussis in the community. It is worth noting that the proportion of notifications that were laboratory confirmed, in particular by serology, did not increase between epidemics.

Periodic epidemics of pertussis, on a background of endemic disease, continue to occur despite high rates of vaccination for pertussis in children. In June 2001, reported uptake in NSW of the first three doses of pertussis vaccine was approximately 91 per cent, and for the 18-month-dose was 88 per cent. ${ }^{5}$ As of 31 December 2002 , reported uptake has increased slightly to 93 per cent for the primary series and 91 per cent for the 18 month dose. ${ }^{6}$ The effectiveness of three or more doses of pertussis whole-cell vaccine in NSW children has been estimated as 91 per cent in children under two years of age, 85 per cent in children aged 2-4 years and 87 per cent in those aged 5-8 years. ${ }^{7}$ While infants are comprising a smaller percentage of total notifications in each epidemic (those under five years of age made up 21 per cent in 1993-1994, 12 per cent in 1997-1998, and eight per cent in 2000-2001), notification rates in young infants (one year of age and under) have not fallen substantially.

Our data suggest that waning immunity has resulted in a cohort of individuals susceptible to pertussis. It is the same cohort of children in 1997 and 2000-2002 that was maximally affected (that is, children who were born before 1990 and aged 8-9 years in 1997). This cohort of children was the last cohort not to have received a booster dose of pertussis vaccine at age four years. This booster dose was added to the routine childhood immunisation schedule in 1994. It is now over 10 years since this cohort received their last immunisation, routinely scheduled for 18 months of age. It is likely that immunisation uptake of the primary course and the 18-month dose was also suboptimal: the 1989-1990 National Health Survey found that in NSW only 70.2 per cent of children aged 0-6 years were fully immunised against pertussis. ${ }^{8}$

Unlike the existing pertussis vaccine, which is recommended for use only in children aged eight years and under, the newly available adult pertussis vaccine is suitable for use in young adolescents, such as the cohort maximally affected in the NSW epidemic.

\section{BOX 2}

\section{DRAFT NATIONAL RECOMMENDATIONS FOR IMMUNISATION AGAINST PERTUSSIS}

(proposed in the draft eighth edition of the Australian Immunisation Handbook) ${ }^{9}$

- cease the 18-month dose, given the prolonged immunity resulting from a primary course of DTPa, with the fourth dose now due at four years of age; replace the existing recommendation for ADT at age 15-17 years with adult acellular pertussis (combined with tetanus and diphtheria antigens) vaccine (dTPa);

- recommend a booster dose before planning pregnancy, or for both parents as soon as possible after delivery of an infant;

- recommend a booster dose for adults working with young children, especially health care workers and child-care workers in contact with young infants;

- encourage receipt of a booster dose in any adult expressing an interest;

- subsequent boosters are not recommended.

The proposed strategy for reducing infant exposure to pertussis recognises that targeting new parents, health care workers, and child-care workers for immunisation may be more effective than a more general approach (see Box 2). In the absence of sustained immunity following either vaccination or infection, pertussis is not a candidate for elimination. Modelling of the likely impact of the adult vaccine, if it was provided to the whole adult population every 10 years, predicts only very small benefits in terms of reduced exposure of young infants. ${ }^{10}$

While increasing awareness of pertussis in adults and the widespread use of serology for testing in NSW may explain the apparent increase in notifications in adults, the occurrence of adult pertussis is certainly not new. In 1884 , British physicians commented about pertussis:

'The disease is most common in childhood but is not confined to that period of life. The phenomenon of an old person suffering from whooping cough is far from uncommon. ${ }^{11}$

Our new evidence of the extent to which adults are affected, however, serves to underscore the ongoing challenges that we face in 2003 in trying to reduce mortality and morbidity from pertussis. 


\section{REFERENCES}

1. National Health and Medical Research Council. The Australian Immunisation Handbook, 7th edition. Canberra: National Health and Medical Research Council, 2000.

2. Rea MD, Dalton CB, Ebeling PW, Ferguson JK. Pertussis death in the Hunter region of New South Wales [letter]. Med J Aust 2001;175:172-3.

3. Halperin SA, Bortolussi R, Langley JM, Miller B, Eastwood BJ. Seven days of erythromycin estolate is as effective as fourteen days for the treatment of Bordetella pertussis infections. Pediatrics 1997;100:65-71.

4. Poynten IM, Hanlon M, Irwig L, Gilbert GL. Serological diagnosis of pertussis: evaluation of $\operatorname{IgA}$ against whole-cell and specific Bordetella pertussis antigens as markers of recent infection. Epidemiol Infection 2002;128:161-7.

5. Australian Childhood Immunisation Register. Health Insurance Commission, Canberra. Date of processing 30 June 2001.

6. Australian Childhood Immunisation Register. Health Insurance Commission, Canberra. Date of processing 31 December 2001.
7. Torvaldsen S, Simpson JM, McIntyre PB. Effectiveness of pertussis vaccination in New South Wales, Australia, 19968. Eur J Epidemiol 2003;18:63-9.

8. Australian Bureau of Statistics. 1989-90 National Health Survey Children's immunisation, Australia. Catalogue no 4379.0. Release data 9 October 1992. Canberra: Australian Government Printer, 1992.

9. Australian Technical Advisory Group on Immunisation. Draft 8th edition Australian Immunisation Handbook. Canberra: Commonwealth Department of Health and Ageing, October 2002.

10. Hethcote HW. Simulations of pertussis epidemiology in the United States: effects of adult booster vaccinations. Mathematical Biosciences 1999;158:47-73.

11. Physicians and Surgeons of the Principal London Hospital. The Family Physician. Manual of Domestic Medicine. Volume 1, 1884.

\section{DO VARIATIONS IN PERTUSSIS NOTIFICATIONS REFLECT INCIDENCE OR SURVEILLANCE PRACTICES? A COMPARISON OF INFANT NOTIFICATION RATES AND HOSPITALISATION DATA IN NSW}

\author{
Siranda Torvaldsen and Peter McIntyre \\ National Centre for Immunisation Research and \\ Surveillance of Vaccine Preventable Diseases \\ University of Sydney, Westmead
}

The incidence of pertussis cannot be directly measured; estimates are generally based on data sources such as notifications, hospitalisations and deaths. However, these data represent only a proportion of the total cases occurring in the community. The accuracy of notification data may vary between states and territories or over time due to different surveillance practices and as new diagnostic tests are introduced. These surveillance issues, and the typical three-to-five-year cycles of pertussis epidemics, make comparisons over time and between states and territories difficult. However, the comparisons should be more valid for hospitalisation data than for notification data, because methods of collecting hospitalisation data are likely to be more uniform. Some variation in incidence by geographical area is to be expected, depending on the timing of the last epidemic and also on geographical differences in past or present pertussis vaccination coverage. If geographical variations in pertussis notifications reflect real variations in pertussis incidence rather than reporting differences, areas with high infant (aged less than 12 months of age) notification rates would be expected to have high infant hospitalisation rates. This article describes and compares the notification and hospitalisation rates in infants for pertussis, by health area, over a period incorporating two pertussis epidemics in NSW.

\section{METHODS}

Medical practitioners and laboratories in NSW are required by legislation to notify the NSW Department of Health, through one of the 17 public health units, of any person who meets the case definition for pertussis. This information is then entered into the Notifiable Diseases Database (NDD). The case definition for pertussis is: ${ }^{1}$

- isolation of Bordetella pertussis from a clinical specimen; or

- elevated Bordetella pertussis specific IgA in serum or Bordetella pertussis antigen in a nasopharyngeal specimen using immunofluorescence, with a history of clinically compatible illness; or

- an illness lasting two weeks or more with one of the following: paroxysms of coughing, inspiratory 'whoop' without other apparent causes, or post-tussive vomiting; or 